\title{
Ancestral reconstruction of reproductive traits shows no tendency toward terrestriality in leptodactyline frogs
}

Elisa Barreto Pereira ${ }^{1,2^{*}}$, Rosane Garcia Collevatti ${ }^{1}$, Marcelo Nogueira de Carvalho Kokubum ${ }^{3}$, Núbia Esther de Oliveira Miranda ${ }^{1}$ and Natan Medeiros Maciel ${ }^{1,2}$

\begin{abstract}
Background: Traditionally, the evolution of terrestrial reproduction in anurans from ancestors that bred in water has been accepted in the literature. Still, the existence of intermediate stages of water dependency, such as species that lay eggs close to water (e.g., in burrows) instead of in bodies of water, supports the hypothesis of an ordered and gradual evolution in the direction of a more terrestrial form of reproduction. However, this conventional view has recently been challenged for some anurans groups. Leptodactylinae frogs are a remarkable example of anurans with an outstanding diversity in terms of reproductive features, with distinct water dependency among lineages. Here, we tested the hypothesis of a gradual and ordered tendency towards terrestriality in Leptodactylinae, including the existence of obligatory intermediate stages, such as semi-terrestrial reproductive strategies. We also addressed the association between reproductive modes and the morphological and ecological features.
\end{abstract}

Results: An ancestral reconstruction analysis indicated that even though shifts from aquatic to terrestrial breeding occurred throughout the history of Leptodactylus and Adenomera, shifts from terrestrial to aquatic reproduction happened at almost the same frequency. Our results also demonstrated that reproductive modes for semi-terrestrial tadpoles were not necessarily an intermediate form between aquatic and terrestrial breeds. Correlations among reproductive modes and other life-history traits suggested that tadpole environment, clutch size, nuptial spines, and egg pigmentation were co-evolving and driven by water dependency.

Conclusions: Our results found no evidence of evolutionary tendencies toward terrestriality in Leptodactylinae. We found reversals from terrestrial to aquatic tadpole development and no evidence of obligatory intermediate stages, such as semi-terrestrial reproductive strategies. We also found correlations between reproductive modes and other life-history traits driven by water dependence. Aquatic reproductive modes are associated with higher clutch sizes, lentic waters, and the presence of nuptial spines and egg pigmentation.

Keywords: Adenomera, Leptodactylus, Reproductive mode, Stochastic inference, Trait correlation

\section{Background}

The evolution of life-history traits in different lineages is a major question in the field of evolutionary biology [1], especially because traits may drive the speciation process

\footnotetext{
*Correspondence: elisabpereira@gmail.com

'Laboratório de Genética \& Biodiversidade, Instituto de Ciências Biológicas, Universidade Federal de Goiás (UFG), Campus Samambaia, 74001-970 Goiânia, Goiás, Brazil

${ }^{2}$ Laboratório de Herpetologia e Comportamento Animal Departamento de Ecologia, Instituto de Ciências Biológicas, Universidade Federal de Goiás,

Campus Samambaia, 74001-970 Goiânia, Goiás, Brazil

Full list of author information is available at the end of the article
}

[2,3]. Indeed, niche-related traits, for example, could diverge, producing reproductive isolation between two populations. As a result, this leads to ecological speciation. Coevolution is another interesting topic that determines the process of evolutionary change in a trait triggered by other trait in a lineage. Trait evolution among lineages could occur through reciprocal evolution between interacting species, driven by natural selection [4].

Analyses of character evolutionary history, such as ancestral state reconstruction (reviewed by [5]) and stochastic character mapping [6], are powerful methods for studying 
the origin and maintenance of phenotypic diversity [2,3]. Moreover, the analysis of character associations may provide important clues regarding the coevolution between life-history traits [7]. The Bayesian approach to investigating patterns of ancestral states is considered a more powerful method compared to other methods, such as parsimony. Parsimony is unable to consider more than a single change along the branch on a cladogram and cannot couple with evolutionary time and amount of character change [8].

In amphibians, the evolution of reproductive features is still not completely understood due to the remarkable diversity of lifestyles, from purely aquatic to arboreal and fossorial [9]. Anuran reproductive features are classified by reproductive modes based on oviposition, development, stage and size of hatchling, and parental care $[9,10]$. Each reproductive mode classification in frogs is assigned a number according to the dependence on water for reproduction. For instance, mode number 1 is associated with frogs that deposit their eggs in water where exotrophic tadpoles develop. Frogs presenting reproductive mode number 17 lay eggs in excavated nests where tadpoles live in early stages and subsequently complete their development in ponds or streams. Direct development frogs present reproductive mode number 23 (see details in [9]).

Shifts from aquatic to terrestrial breeding have occurred repeatedly and independently in many vertebrates [11]. Traditional knowledge claims that the evolution of terrestrial reproduction in anurans occurred from ancestors that bred in water $[10,12]$, especially because the aquatic mode is the most representative of exotrophic tadpoles [9] and was probably the ancestral state for anurans [13]. In addition, the existence of intermediate stages of water dependency, such as species that lay eggs close to water (e.g., in burrows) instead of inside bodies of water, supports the hypothesis of an ordered and gradual evolution in the direction of a more terrestrial reproduction $[12,14]$. Nonetheless, Gomez-Mestre et al. [13] has recently challenged this conventional view by demonstrating the lack of intermediate stages in some groups, as well as the evolution of direct development from both terrestrial and aquatic reproductive modes.

In addition, changes between aquatic and terrestrial breeding may occur in conjunction with modifications of morphological and other ecological features [15,16], providing opportunities for coevolution between traits. Even though reproductive modes are frequently studied, the only well-known associations that are commonly tested show: (i) negative correlations between ovum and clutch size (number of eggs per spawning); (ii) positive correlations between ovum size and hatchling dimensions; and (iii) positive correlations between clutch volume, egg size and female body size within a given reproductive mode (see [12]). Under a cladistic perspective, a recent study shed light on some unexplored associations, such as the correlation of terrestrial reproduction with reduced clutch and adult size, and with parental care [13]. However, little is known about other traits that may be correlated with reproductive modes, such as tadpole and adult morphological characteristics, with the exception of body size.

Amphibian systematics has undergone pronounced changes over the past decade (e.g., $[17,18])$. The genus Leptodactylus, the most diverse of the Leptodactylidae, contains 75 species distributed throughout North America (southern Texas), as well as Central and South America. Formerly, the genus was assembled in five groups based on behavioral, morphological and ecological features [15]: the Leptodactylus ocellatus group, now referred to as the L. latrans group [19], the L. melanonotus group, the L. pentadactylus group, the L. fuscus group and the $L$. marmoratus group. However, since Heyer's [15] suggestion that the group Leptodactylus marmoratus was not closely related to the other groups, the phylogenetic position of the group has been discussed, leading to its placement in a different genus, Adenomera [20]. Recent molecular data confirm Adenomera as a natural group with a single common ancestor $[18,21]$. Distributed throughout almost all of South America, the genus is currently comprised of 18 species. This number is known to be underestimated, however, due to the occurrence of cryptic species [22,23].

Leptodactylus and Adenomera (Anura: Leptodactylidae: Leptodactylinae) are good models for understanding the patterns and processes of the evolutionary history of reproductive traits. These foam-nesting species present at least four different reproductive modes, varying in oviposition and biology of the larvae. The diversity of reproductive modes for Leptodactylus and Adenomera, as well as its association with species in the phenetic groups of Leptodactylus and in the Adenomera, has led to the prediction of a gradual evolutionary tendency of evolutionary lineages, from a more aquatic to a more terrestrial breeding. This also suggests the presence of obligatory intermediate stages of water dependence to reproduction. Heyer [15] hypothesized that the Leptodactylus melanonotus and L. latrans groups had the most primitive reproductive modes, with higher water reliance (Figure 1, mode number 11). The Leptodactylus pentadactylus group would represent the first step towards terrestriality, with eggs placed in the water accumulated in basins constructed by males (Figure 1, mode number 13), followed by the L. fuscus group, in which eggs are placed inside subterranean chambers that are also constructed by males (Figure 1, mode number 30 ). Finally, Adenomera (formerly the L. marmoratus group) would represent the most derived reproductive mode, 


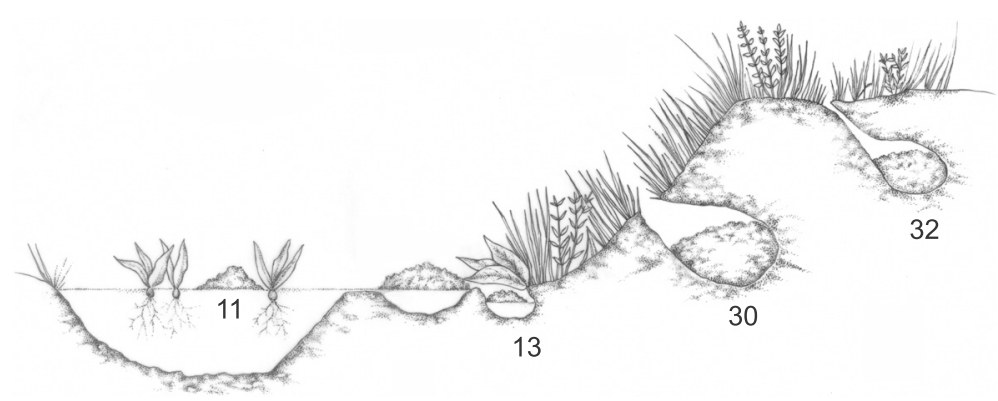

Figure 1 Schematic drawings representing known reproductive modes in Leptodactylinae. Mode 11 includes species that produce floating foam nests in ponds with exotrophic tadpoles; Mode 13 also presents exotrophic tadpoles, but with foam nests placed in water accumulated in constructed basins; Mode 30 groups species that have foam nests that are placed inside a subterranean chamber and after a period of development, the tadpoles float to the bodies of water; Mode 32 is the most terrestrial one, with endotrophic tadpoles (developing entirely in subterranean chambers using only the yolk as a source of energy). Illustrated by Vinícius Yano.

with a lower dependency on water for reproduction, since some species are known to have endotrophic tadpole development in subterranean chambers constructed by males (Figure 1, mode number 32). However, Heyer [15] suggested that Adenomera was an independent lineage, and postulated that the evolutionary shift to terrestrial reproduction in leptodactylines occurred twice, once in the Leptodactylus ancestor and another in the Adenomera ancestor. Although this hypothesis of a gradual increase of terrestriality in some Leptodactylinae frogs has never being tested, it has repeatedly been cited in the literature (e.g., [9,24-28]). Recently, some authors have raised questions concerning this hypothesis $[29,30]$.

Here, we studied the evolution of life-history traits among Leptodactylinae lineages by reconstructing ancestral states, mapping character changes and testing the correlation among six characteristics. In this study, we tested: (1) the hypothesis of the tendency towards terrestriality, with shifts from aquatic to terrestrial breeding and the existence of obligatory intermediate stages; and (2) the association between reproductive modes and morphological and ecological features that are potentially related to water dependency.

\section{Results}

Phylogeny estimation

The combined dataset alignment consisted of a fragment of 1,526 base pairs (Table 1). The third codon position of $c y t B$ was excluded from the final alignment due to high saturation (Additional file 1). Some hypervariable regions with several indels were excluded from the $12 \mathrm{~S}$ and $16 \mathrm{~S}$ sequences because of the ambiguous alignments they generated. For both the $12 \mathrm{~S}$ and $16 \mathrm{~S}$ datasets, the best evolutionary model was $\mathrm{GTR}+\mathrm{I}+\mathrm{G}$, whereas for the $c y t B$ fragment, it was TIM $2+\mathrm{I}+\mathrm{G}$. Finally, for the $R$ hod fragment, the best evolutionary model was TPM3uf $+\mathrm{I}+\mathrm{G}$ (Table 1).

The Bayesian analysis resulted in a monophyletic and highly supported Adenomera clade with Lithodytes lineatus as a sister species (Figure 2). Adenomera heyeri and A. lutzi comprises a sister clade of all other Adenomera species sampled. The Leptodactylus species also formed a highly supported monophyletic group, subdivided into two major clades (Figure 2).

The maximum parsimony analyses produced 50 of the most parsimonious trees with 1,435 steps. The strict

Table 1 Sequence characterization and evolutionary model used in phylogenetic analyses for 35 Leptodactylinae species

\begin{tabular}{|c|c|c|c|c|}
\hline & $16 S$ & $12 S$ & Cytochrome B & Rhodopsin 1 \\
\hline Original length (bp) & 517 & 435 & 405 & 330 \\
\hline Final length (bp) & 503 & 423 & 270 & 330 \\
\hline \multicolumn{5}{|l|}{ Base frequencies } \\
\hline$\% A$ & 0.314 & 0.308 & 0.211 & 0.234 \\
\hline$\% \mathrm{C}$ & 0.230 & 0.262 & 0.216 & 0.283 \\
\hline$\% G$ & 0.210 & 0.203 & 0.230 & 0.195 \\
\hline$\% \mathrm{~T}$ & 0.246 & 0.227 & 0.343 & 0.288 \\
\hline Parsimony informative characters (PIC) & 116 & 107 & 28 & 44 \\
\hline PIC without outgroup & 108 & 101 & 27 & 27 \\
\hline Best fit model & $\mathrm{GTR}+\mathrm{I}+\mathrm{G}$ & $\mathrm{GTR}+\mathrm{I}+\mathrm{G}$ & $\mathrm{TIM} 2+\mathrm{I}+\mathrm{G}$ & TPM3uf $+1+G$ \\
\hline Model likelihood & $2,904.84$ & $2,865.12$ & 868.58 & $1,081.27$ \\
\hline
\end{tabular}




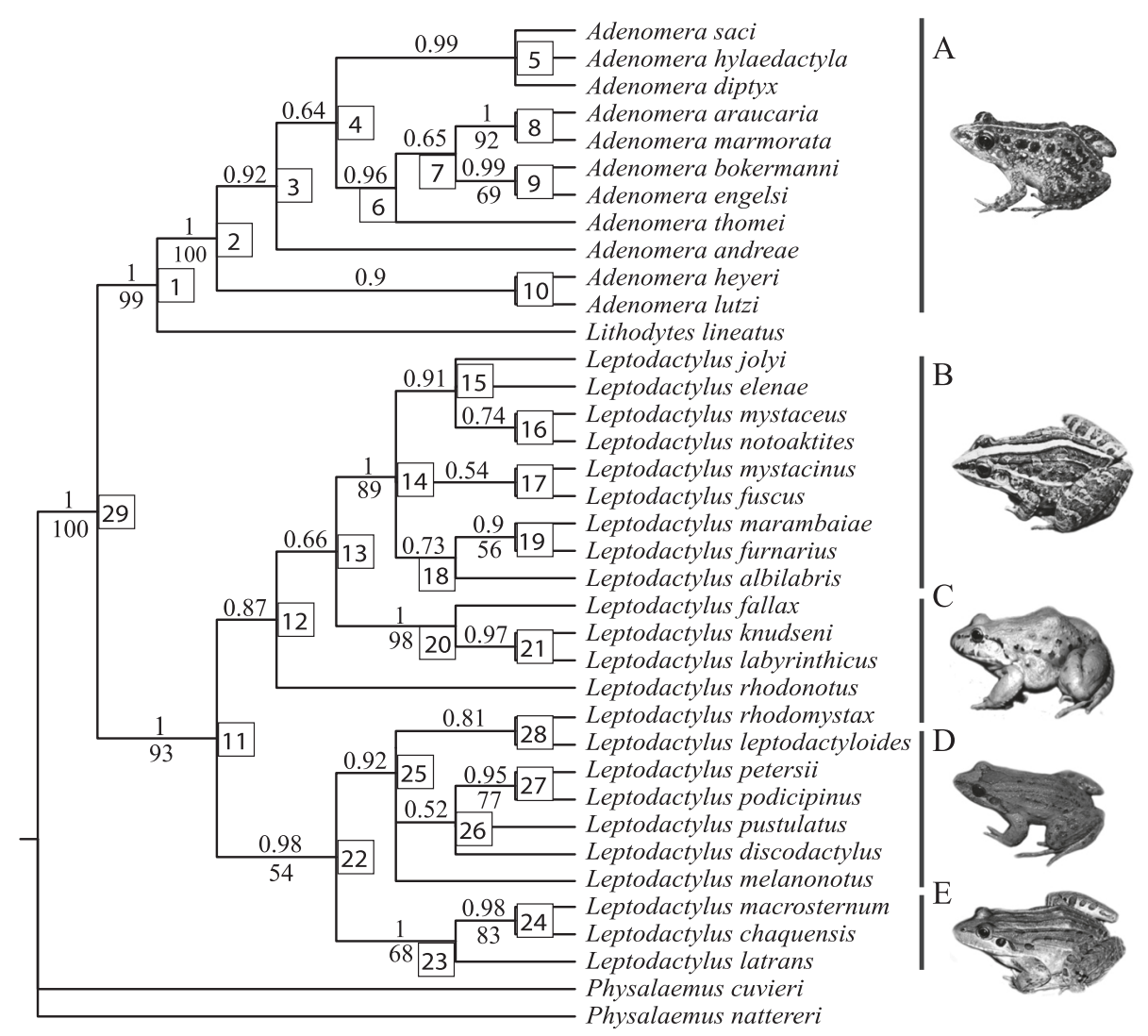

Figure 2 Phylogenetic relationships among Leptodactylinae, based on the 50\% majority rule consensus cladogram reconstructed using the Bayesian analysis. Numbers inside squares represent clade numbers. Numbers above nodes are clade posteriori probability, and below nodes are bootstrap supports for the maximum parsimony analysis. A: Adenomera saci (Adenomera genus); B: Leptodactylus fuscus (L. fuscus group); C: L. labyrinthicus (L. pentadactylus group); D: L. podicipinus (L. melanonotus group); and E: L. latrans (L. latrans group). Photos: A, Pedro Peloso, B and D, Ariovaldo Giaretta, and $C$ and $E$, Antonio Sebben.

consensus tree had 1,472 steps $(\mathrm{CI}=0.41, \mathrm{RI}=0.56)$ and also showed Lithodytes lineatus as a sister species to the monophyletic genus Adenomera. The parsimony analyses generated a consensus tree similar to the Bayesian analysis, but also presented some polytomies.

\section{Ancestral state reconstruction, character mapping, and correlation}

The Bayesian character state reconstruction indicated that reproductive mode 11, in which eggs are placed in floating foam nests directly on the top of water, was the most probable ancestral state of the most recent common ancestor of Leptodactylinae (node 29, Figure 3). This reproductive mode was also the inferred ancestral state of Lithodytes + Adenomera (node 1) and of Leptodactylus (node 11). While reproductive mode 11 had one origin, modes 13 and 32 originated twice and mode 30 originated at least three times (Figure 3). Reproductive mode 13 is characterized by the deposition of foam nests on water that has accumulated in a constructed basin. In mode 30, parents produce foam nests where eggs and early larval stages develop in subterranean constructed nests. Subsequently, exotrophic tadpoles finish their development in ponds. Similar to mode 30, species with reproductive mode 32 reproduce in subterranean constructed chambers. However, the endotrophic tadpoles complete their development in a nest.

In addition, the analysis showed that transitions from aquatic to terrestrial (or at least to less aquatic) reproductive modes have occurred at least four times in Leptodactylinae: 1) a shift from mode 11 to modes 30 or 32 in the ancestral Adenomera (nodes 1 and 2, Figures 2 and 3); 2) a shift from mode 30 to 32 in some Adenomera species (nodes 6 and 7); 3) a shift from mode 11 to 30 in some Leptodactylus ancestral (nodes 11 and 12); and a shift from 11 to 13 in two species of the L. melanonotus group (nodes 26 and 27). Moreover, transitions from terrestrial to aquatic (or at least a less terrestrial) reproductive modes were also found: 1) a shift from mode 32 to 30 in some Adenomera species (nodes 3 and 4); and 2) a shift from mode 30 to 13 in species of the Leptodactylus pentadactylus group (nodes 13 and 20). Besides 


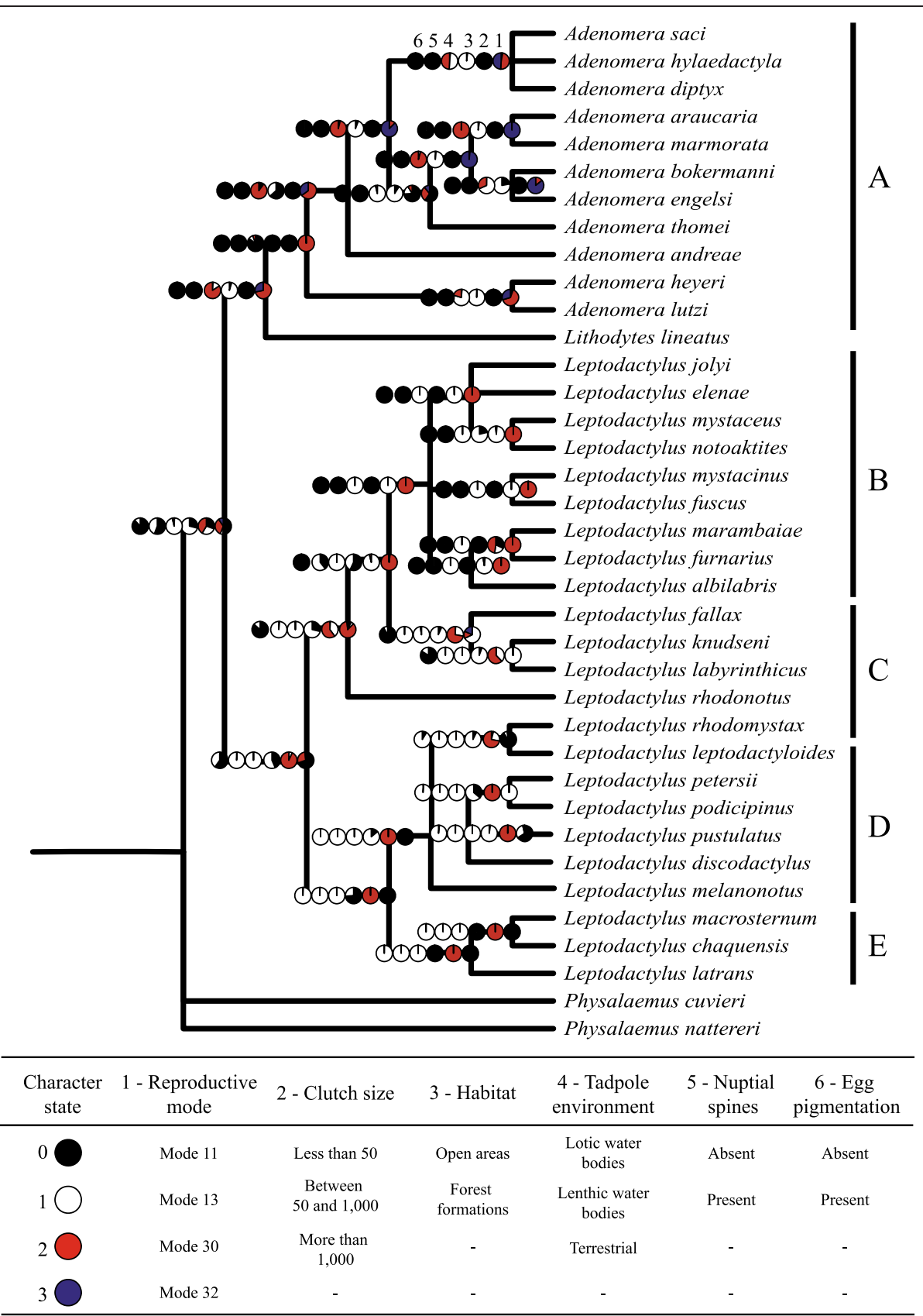

Figure 3 Ancestral state representation of six life-history traits reconstructed for 35 Leptodactylinae species using stochastic inferences. Pie charts indicate the probability of each character state. Clade numbers are indicated at the nodes (inside squares) of the Bayesian cladogram (Figure 2). Only the ancestral state probability of the clades in the 50\% majority-rule consensus cladogram reconstructed using Bayesian analysis are indicated here. See Additional file 2 for the probability of each character state in each possible clade, as pointed out by the Bayesian analysis. A: Adenomera genus; B: Leptodactylus fuscus group; C: L. pentadactylus group; D: L. melanonotus group; and E: L. latrans group.

those, transformation direction in node 2 was uncertain because it had equal probability for reproductive modes 30 and 32 (node 2, see Figure 3). Thus, the reproductive mode may have changed from 30 to 32 in node 3 or from 32 to 30 in node 10 (Figure 3).

While clutch size, tadpole environment, nuptial spines and egg pigmentations presented a clear evolutionary pattern with few independent origins of states (Figure 3), multiple reversals between 'open areas' and 'forest formations' were found by the habitat reconstruction. The most recent common ancestor of Adenomera and Lithodytes (node 1) produced less than 50 eggs per clutch and lacked nuptial spines and melanin on eggs, while the Leptodactylus most recent common ancestor (node 11) 
had large clutch sizes, tadpoles in lentic water bodies, presence of nuptial spines and the absence of egg pigmentation.

The character mapping analysis retrieved the estimated number of changes in the ancestral nodes, together with the probable transformations along the branches (Table 2). The habitat had the highest expected number of changes (approximately 32), being almost the same number from one state to another. The reproductive mode had 20 changes, most of them between modes 32 to 30 . However, nuptial spine presented the lowest number of transformations (eight changes).

The D statistics found significant correlations only between reproductive mode and clutch size or nuptial spines (Table 3). However, significant correlations between specific reproductive modes and other character states were also found (dij statistics; Table 3). For example, although reproductive mode and egg pigmentation presented no significant correlation ( $\mathrm{D}=0.47, \mathrm{p}=0.01$ ), egg pigmentation had a positive association with reproductive modes 11 and 13 (respectively $d i j=0.09, \mathrm{p}=1.0 \mathrm{e}-7$ and 0.02 , $\mathrm{p}=1.0 \mathrm{e}-7)$, and a negative association with mode 32 (dij $=-0.05, \mathrm{p}=1.0 \mathrm{e}-7)$.

\section{Discussion}

\section{Ancestral state reconstruction}

Our results showed that the evolution of reproductive modes in Leptodactylinae did not follow a linear trend, as Heyer [15] predicted, and it did not necessarily happen through intermediate stages, as McDiarmid [14] suggested. Other interesting facts demonstrated here include the monophyletic independent sister lineages of Adenomera and Leptodactylus $[17,18,20,23,31,32]$ and the independent origins of less aquatic modes, occurring in the ancestors of both genus, as suggested by Heyer [15].

Moreover, we found no indication of a gradual evolution of reproductive traits towards terrestriality through the lineages. Transitions were found from the most aquatic mode (11) to the semi-aquatic mode (30) and to the terrestrial mode (32) - both consists in eggs in foam nests deposited in burrows, but while in the first the tadpoles are carried by water to the water body, in the second the tadpoles develops entirely into the burrow. This same situation was demonstrated for other terrestrial modes of reproduction in anurans [13]. This shift from mode 11 to 30 may have happened two times: 1) from the Leptodactylus genus ancestor to the ancestor of the L. fuscus and L. pentadactylus groups; and 2) from the Lithodytes and Adenomera ancestor to the most recent common ancestor of the latter genus. In this last case, even with the confident phylogenetic position of Lithodytes lineatus (which was also shown by $[17,18,21,31,32]$ ), there is still uncertainty about its ancestor state (Figure 2).
Its breeding site is uncommon in the Leptodactylinae species, with the parent laying eggs in foam nests inside ant nests. Besides, it is not clear whether or not the tadpoles subsequently complete their development in temporary water bodies. This reproductive mode does not fit any of those previously assigned to the subfamily.

The absence of a linear evolution from aquatic to terrestrial reproductive modes is confirmed by reversions from mode 30 - eggs in foam nests inside burrows and tadpoles subsequently carried to the water body - to mode 13 - eggs in foam nests in constructed basins near the water - and also from mode 32 - eggs in foam nests and complete development of the tadpoles inside burrows - to mode 30 (Figure 2). Reversions from terrestrial to aquatic reproductive modes have also been demonstrated in other anuran groups [13]. Also, the character mapping analysis showed that these transitions occurred not only at the most recent common hypothetic ancestor (at the nodes), but also along the branches. The transitions from a more terrestrial breeding mode to one that is less-so can also be observed by summing up the expected number of transformations in this direction and comparing them to the transformations from aquatic to terrestrial breeding (see Table 2). Both presented nearly 10 transformations, showing that terrestrial egg laying is not necessarily an evolutionary tendency, but is actually an alternative strategy with no implied directionality.

The analysis of ancestral state reconstruction of clutch size also showed evidence of a clear pattern among lineages. The ancestral state of the Leptodactylus species suggested the oviposition of more than 1,000 eggs per clutch, while Adenomera had less than 50 eggs (see Figure 3). Even though a reversion was found from in Adenomera from the reproductive mode which tadpoles develops inside the burrows (32) to the mode that tadpoles are carried from the burrow to the water body (30), the hypothetical ancestral state holds the oviposition of few eggs. It is unlikely that the exotrophic Adenomera tadpoles simply float to the water bodies by chance (e.g., due to topography or great rain incidence), because they have functional mouthparts and spiracles [33,34], implying that they need nourishment provided from the environment to complete their metamorphoses. Endotrophic tadpoles do not have mouthparts or spiracles, as they use only the yolk provided by their parents for their development.

\section{Character mapping and correlations}

A clear association pattern between clutch size and reproductive mode was found, as predicted by Heyer [15] and previously demonstrated for many anuran genera [13]. The higher dependence on water, which is a more unpredictable environment when compared to a burrow, together with no parental care, favors r-strategists, which 
Table 2 Evolutionary data for six Leptodactylinae life-history traits based on stochastic Bayesian character mapping

\begin{tabular}{|c|c|c|c|c|c|c|c|c|c|c|c|c|c|c|c|c|c|c|c|}
\hline \multirow[t]{2}{*}{ Character } & \multirow[t]{2}{*}{ Replications } & \multirow{2}{*}{$\begin{array}{l}\text { Expected } \\
\text { number of } \\
\text { transformations }\end{array}$} & \multicolumn{12}{|c|}{ Expected number of character state transformation } & \multicolumn{4}{|c|}{ Amount of time } & \multirow[t]{2}{*}{ Rate } \\
\hline & & & $0-1$ & $0-2$ & $0-3$ & $1-0$ & $1-2$ & $1-3$ & $2-0$ & $2-1$ & $2-3$ & $3-0$ & $3-1$ & $3-2$ & State 0 & State 1 & State 2 & State 3 & \\
\hline Reproductive mode & 60,000 & 19.87 & 2.53 & 2.6 & 1.1 & 1.04 & 1.3 & 0.5 & 1.9 & 1.4 & 1.8 & 1 & 0.6 & 4.1 & 0.30 & 0.16 & 0.32 & 0.22 & 8.16 \\
\hline Clutch size & 60,000 & 14.30 & 1.78 & 1.9 & - & 1.72 & 2.6 & - & 2.3 & 4 & - & - & - & - & 0.33 & 0.31 & 0.36 & - & 5.62 \\
\hline Habitat & 60,000 & 31.60 & 16.4 & - & - & 15.2 & - & - & - & - & - & - & - & - & 0.46 & 0.54 & - & - & 11.8 \\
\hline Tadpole environment & 60,000 & 16.39 & 1.83 & 1 & - & 2.66 & 4.8 & - & 1.5 & 4.6 & - & - & - & - & 0.08 & 0.69 & 0.23 & - & 6.33 \\
\hline Nuptial spines & 60,000 & 7.94 & 2.91 & - & - & 5.03 & - & - & - & - & - & - & - & - & 0.58 & 0.42 & - & - & 2.64 \\
\hline Egg pigmentation & 60,000 & 13.10 & 6.25 & - & - & 6.85 & - & - & - & - & - & - & - & - & 0.69 & 0.31 & - & - & 4.37 \\
\hline
\end{tabular}

Estimated number of state transformations, amount of time and rate of transformation for each character for the 35 Leptodactylinae species based on stochastic Bayesian character mapping using 600 trees (See characters codes in Table 4). 
Table 3 Correlation values obtained by the $\mathrm{D}$ and dij statistic for 35 Leptodactylinae species.

\begin{tabular}{|c|c|c|c|c|c|c|}
\hline & \multirow{2}{*}{$\begin{array}{l}\text { Reproductive mode } \\
D\end{array}$} & & \multicolumn{4}{|c|}{ Reproductive mode $d i j$} \\
\hline & & & Mode 11 & Mode 13 & Mode 30 & Mode32 \\
\hline \multirow[t]{3}{*}{ Clutch size } & 0.72 & Less than 50 & -0.07 & -0.04 & -0.01 & 0.12 \\
\hline & & Between 50 and 1,000 & -0.03 & -0.01 & 0.08 & -0.05 \\
\hline & & More than 1,000 & 0.09 & 0.04 & -0.07 & -0.07 \\
\hline \multirow[t]{2}{*}{ Habitat } & 0.30 & Open areas & -0.01 & -0.02 & 0.06 & -0.03 \\
\hline & & Forest formations & 0.01 & 0.02 & -0.06 & 0.03 \\
\hline \multirow[t]{3}{*}{ Tadpole environment } & 0.49 & Lotic water bodies & -0.02 & -0.01 & 0.02 & - \\
\hline & & Lentic water bodies & 0.07 & 0.03 & 0.01 & - \\
\hline & & Terrestrial & - & - & - & - \\
\hline \multirow[t]{2}{*}{ Nuptial spines } & 0.65 & Absent & -0.10 & -0.06 & 0.08 & 0.08 \\
\hline & & Present & 0.10 & 0.06 & -0.08 & -0.08 \\
\hline \multirow[t]{2}{*}{ Egg pigmentation } & 0.47 & Absent & -0.09 & -0.02 & 0.06 & 0.05 \\
\hline & & Present & 0.09 & 0.02 & -0.06 & -0.05 \\
\hline
\end{tabular}

Bolded values indicate $p \leq 0.05$.

may allocate energy to increase the number of eggs per clutch. Conversely, species with a low dependency on water, in this case, egg-burrowing species, may allocate energy to parental care [35,36]. The correlation analyses showed that the ancestral state of Leptodactylus and Adenomera species presents both kinds of reproductive strategies, with the first favoring productivity, and the other favoring parental care.

The construction of the subterranean chambers by males of the Leptodactylus fuscus group and the Adenomera species is considered a type of parental care, with the parents providing a more suitable microhabitat for offspring development $[14,37]$. When compared to water environments, subterranean chambers increase the chance of offspring survivorship by reducing predation, desiccation and interspecific competition [14,38,39]. Egg-burrowing species have a limitation regarding the number of eggs due to both the amount of energy spent in the burrow construction, and the limited space inside the chamber [40]. Thus, space may be an important constraint, since terrestriality in anurans demands increased amounts of yolk to feed the endotrophic tadpoles, consequently increasing egg dimensions [15,40,41]. The amount of yolk needed in the reproductive mode which tadpoles complete the development inside the burrow (32) is higher than in mode in which they are carried to the water (30), because in the first case the tadpole completes the development exclusively using yolk as an energy source. Conversely, species with mode 30 only depend on the yolk for a brief period of tadpole development, which may lead to contrasting correlations of modes 32 and 30 and clutch sizes. An opposite relationship was noticed in the more aquatic reproductive mode, with tadpoles using external sources of energy from the beginning of development, leading to a smaller egg dimension and larger clutch size, and consequently, to a correlation of egg size and oviposition.

We found no evidence of a clear association with the phylogenetic hypothesis of Leptodactylinae and evolution in their habitat usage (open and forest formations), especially since this trait presented the highest number of expected transformations and no significant correlations with reproductive modes. The correlations provide no evidence to support the hypothesis that the evolution of terrestrial breeding is linked to forest habitats due to the high humidity $[42,43]$. However, air humidity may not limit the development of Leptodactylinae because the foam nest may protect eggs from desiccation. Although we are aware that the lack of correlation and the high number of transformations may be a consequence of the small number of categories (open and forest), there is not enough data available to be more specific about the habitat that these species use.

Environmental filters seem to have been decisive for the evolution of tadpole environment in Leptodactylus, since all species shared the same state: lentic water bodies. This tadpole environment appears in Leptodactylinae during almost $70 \%$ of the lineage reconstructions (see Table 2). Species with aquatic reproduction that involves placing eggs in lentic environments have an adaptive advantage when compared to those that use lotic water bodies because lentic waters facilitate the amplexus, providing a sheltered environment for eggs and the tadpole's first stage of development, in addition to keeping the nest integrity. Consequently, the reproductive modes more related to aquatic breeding (11 and 13) are negatively correlated with lotic water bodies.

Our results showed similar evolutionary histories for nuptial spines and egg pigmentation. The presence or absence of these structures occurred together in almost 
the same species and ancestral nodes, with two major exceptions: the ancestor of Leptodactylus and the L. pentadactylus group. Both lacked egg pigmentation but had nuptial spines, which help the male anchor to the female. The presence of nuptial spines in Leptodactylus' ancestors was maintained in the ancestors of the Leptodactylus latrans and L. melanonotus groups, including $L$. discodactylus, but is now associated with the presence of melanin on eggs. Although Leptodactylus discodactylus has not been assigned to any phenetic groups yet, our results suggest that it belongs to the L. melanonotus group (see $[18,21,31])$. The ancestral state of the Leptodactylus pentadactylus group, which had nuptial spines but lacked melanin on eggs, corroborates Heyer's [15] hypothesis. He stated that the presence of spines in the Leptodactylus pentadactylus group has evolved because of the large adult body size of this species rather than because of the water dependence.

The species in this group do not place eggs directly in a main body of water. Spines are used to facilitate the amplexus between large specimens where eggs are placed in water accumulated in basins constructed by males. The lack of egg pigmentation in the group's ancestors, which is only needed in eggs exposed to ultraviolet light, also supports Heyer's hypothesis. Nevertheless, we found a positive correlation between mode 13 (eggs in constructed basins near the water), which is common among the Leptodactylus pentadactylus group and some species of the Leptodactylus melanonotus group, and egg pigmentation. This could be associated with the ability of species in the Leptodactylus pentadactylus and Leptodactylus melanonotus groups to construct basins where eggs are spawned [26]. In many cases, the eggs are exposed to ultraviolet light, and the egg pigmentation helps protect against embryo damage. The correlation analysis also confirmed the association between the presence of spines and egg pigmentation with the more aquatic reproductive mode (mode 11) and a negative correlation with the more terrestrial breeding (mode 32).

\section{Conclusions}

Our results showed no evidence of an evolutionary tendency toward terrestriality in Leptodactylinae. Indeed, we found reversals from terrestrial to aquatic tadpole development and no evidence of mandatory intermediate stages. In addition, we also found correlations between morphological and ecological traits driven by water dependence. Aquatic reproductive modes are associated with higher clutch sizes, lentic waters, and the presence of nuptial spines and egg pigmentation. No correlation was found between reproductive modes and habitat usage, where multiple reversals of ancestors and descendants living in open and forested areas were found. The robustness of the phylogenetic hypothesis, which confirmed Adenomera and
Leptodactylus monophyly and Lithodytes as a sister taxon of Adenomera, enabled the study of reproductive trait evolution. Furthermore, the present study reinforces the usefulness and power of Bayesian stochastic character mapping to better understand the evolution of life history traits.

\section{Methods}

\section{Taxon sampling}

We sampled 35 Leptodactylinae species, 11 Adenomera, and 23 Leptodactylus, representing all recognized phenetic groups: the Leptodactylus latrans group, the Leptodactylus melanonotus group, the Leptodactylus pentadactylus group, and the Leptodactylus fuscus group, as well as the monotypic Lithodytes lineatus (Additional file 3). Physalaemus cuvieri and Physalaemus nattereri were used as outgroups based on their relationships with Leptodactylinae $[17,18]$. Ninety-one sequences were obtained in this work and 40 were obtained from GenBank (Additional file 3).

Although our phylogenetic sampling does not represent the majority of leptodactyline species, we are confident that the results of character reconstruction, character mapping and correlation analyses will hold even if the remaining species were included in the phylogenetic hypothesis because: (i) most species with phylogenetic uncertainty (Adenomera) were included, (ii) these species were also the ones with higher variations in character states regarding reproductive modes; (iii) previous studies pointed out that Leptodactylus phenetic groups are phylogenetically structured, especially the L. fuscus, L. melanonotus (with L. discodactylus), and L. latrans [18,23,31,44], (iv) although the phylogenetic positioning of L. pentadactylus group was not yet certain, our results are in consonance with previous ones $[18,31,32,44-47]$, and (v) most of the reproductive and morphological attributes of Leptodactylus were conserved among the phenetic groups $[44,48]$. In addition, natural history traits are not available for many leptodactyline species, which constrains the analysis of trait evolution.

\section{Genetic data}

Total DNA was extracted from muscle or liver tissue preserved in ethanol and a tissue-storage buffer using the DNeasy Tissue Kit (Qiagen ${ }^{\circ}$ ). We sequenced four DNA fragments. The nuclear Rhodopsin exon I (Rhod) fragment was sequenced using Rhod1A and Rhod1C primers [49]. The mitochondrial regions $12 \mathrm{~S}$ and $16 \mathrm{~S}$ were sequenced using $12 \mathrm{Sa}, 12 \mathrm{Sb}, 16 \mathrm{Sar}$ and $16 \mathrm{Sd}$ [50]. For cytochrome B (cytB), we used MVZ15 [51] and H15149 primers [52] (PCR protocols on Additional file 4). The PCR products were purified using $1.0 \mathrm{U}$ of each enzyme, "shrimp alkaline phosphatase" (SAP) and exonuclease I (EXOI) (Biotech Pharmacon, ASA). Purified PCR products were sequenced in both directions on an 
ABI 3100 automated DNA sequencer (Applied Biosystems, CA) using the DYEnamic ${ }^{\text {TM }}$ ET terminator sequencing kit (GE HealthCare, Sweden), according to the manufacturer's instructions.

\section{Sequence alignment and phylogenetic analyses}

The sequences were edited using SeqScape (v2.1) software and were then aligned in MUSCLE 3.8 [53]. The sequences that were not available were coded as missing data (Additional file 3). Coding sequences were tested for saturation plotting transitions and transversions against TN93 distance [54] using the DAMBE [55] software.

Phylogenetic hypotheses were obtained for the combined datasets using Bayesian and maximum-parsimony methods. Evolutionary model selection was performed using Akaike Information Criterion (AIC) implemented in jModelTest 2 [56]. Then, Bayesian analyses were conducted in MrBayes v.3.1.2 [57] with randomly generated starting trees. Four Markov Chains and four million generations were sufficient to obtain a standard deviation of split frequencies below 0.01 . Trees and parameter values were sampled every 500 generations. After discarding the first 2500 trees ("burn-in") of the two runs, we generated the $50 \%$ majority-rule consensus and calculated the Bayesian credibility values (BC) for each branch. Clades with BC equal to or exceeding $95 \%$ were considered strongly supported [58]. The maximum parsimony (MP) analysis was carried out using PAUP* 4.0 [59]. We used a heuristic search with multiple tree bisection reconnection (TBR) branch swapping. Bootstrap resampling [60] was applied to assess the support for individual clades using 1,000 bootstrap replicates and full heuristic searches with 10 replicates of random stepwise addition and TBR branch swapping. Clades with bootstrap values higher than $75 \%$ were considered well-supported following what indicated by [61].

\section{Ancestral state reconstruction, character mapping, and correlation analysis}

To study the evolution of life-history traits among Leptodactylinae lineages, we inferred ancestral states, mapped character state changes and tested the correlation of six ecological and morphological traits using SIMMAP 1.5 [62]. At least four reproductive modes are known for Leptodactylinae [9]: (i) mode '11' includes species that produce floating foam nests in ponds with exotrophic tadpoles; (ii) mode '13' also presents exotrophic tadpoles, but with foam nests placed in water accumulated in constructed basins; (iii) mode '30' groups species with foam nests placed inside a subterranean chamber, and after a period of development, the tadpoles float to bodies of water; and (iv) mode ' 32 ' is the most terrestrial one, with endotrophic tadpoles (develop entirely in subterranean chambers using only the yolk as a source of energy) (Figure 1). Other life-history traits studied here were considered directly (clutch size, tadpole environment, nuptial spines and egg pigmentation) or indirectly (habitat) related to reproductive modes in frogs. Character states were retrieved from the literature (Additional file 2), based on personal observation or from specialists in the reproductive traits of Neotropical anurans (information by authority), and were coded as shown in Table 4.

We reconstructed the ancestral states of the six characters using Bayesian stochastic character mapping [8] on the 50\% majority-rule consensus tree obtained from the Bayesian phylogenetic analysis (Additional file 5). The analysis evaluated the consistency between character history and character states observed at the tips, before then estimating the posterior probabilities of ancestral states. The results were visualized as pie charts using a function in the R software, developed by Dr. Marion Chartier. We also mapped the changes of character states along the phylogeny to estimate the number of transformations between states [6]. To perform this analysis, we randomly selected 600 trees from the Bayesian phylogenetic analysis after the convergence.

Afterwards we calculated the overall character correlation (D statistic) between reproductive mode and five lifehistory traits (i.e., clutch size, habitat, tadpole environment, nuptial spine, egg pigmentation), and the correlation state-by-state $(d i j)$. For this analysis, we randomly selected 300 trees generated after convergence by the

Table 4 Character codification used in the life-history trait analysis of Leptodactylinae

\begin{tabular}{|c|c|c|c|c|}
\hline Character & State 0 & State 1 & State 2 & State 3 \\
\hline Reproductive mode & Mode 11 & Mode 13 & Mode 30 & Mode 32 \\
\hline Clutch size & Less than 50 & Between 50 and 1,000 & More than 1,000 & - \\
\hline Habitat & Open areas & Forest formations & - & - \\
\hline Tadpole environment & Lotic water bodies & Lentic water bodies & Terrestrial tadpole & - \\
\hline Nuptial spine & Absence & Presence & - & - \\
\hline Egg pigmentation & Absence & Presence & - & - \\
\hline
\end{tabular}

The character codification was used in the ancestral state reconstruction, character mapping and correlation analysis of the six life-history traits for 35 species of Leptodactylinae. Polymorphic data were coded as missing data. 
Bayesian phylogenetic analysis. The dij statistic represents the divergence between the observed and expected association of states $i$ and $j$. The expected association is the product of the marginal probabilities of finding these states $(i$ and $j)$ in the same phylogenetic node [7].

\section{Ethics statement}

The Brazilian Institute for Biodiversity Conservation (Chico Mendes Institute for Biodiversity Conservation - ICMBio) provided a license under the number 021010.002153/05-11 to collect some of the specimens used in this study. Many sample tissues were also obtained by donation of other institutions (see Additional file 3). We confirm that all species used in this work are not endangered or protected species.

\section{Availability of supporting data}

The data sets supporting the results of this article are available in the GenBank repository, http://www.ncbi.nlm. nih.gov/genbank, and can be accessed through the identifier indicated in the Additional file 3. The following data sets supporting the results of this article are available in the Dryad Digital repository, [http://dx.doi.org/10.5061/ dryad.88kj7]: 1 . Table containing the GenBank accession numbers of the sequences from Adenomera, Leptodactylus and Lithodytes used in phylogenetic. 2. Table with the character states for the six life-history traits (reproductive mode, clutch size, habitat, tadpole environment, nuptial pads or spines and egg pigmentation) for 35 Leptodactylinae species. 3. State probability of the six reconstructed life-history traits at each ancestral node of the $50 \%$ majority-rule consensus cladogram. 4. Lifehistory traits reconstructed were reproductive mode, clutch size, habitat, tadpole environment, nuptial pads or spines and egg pigmentation. 5. Nexus file used in MrBayes software to generate Bayesian molecular phylogenetic hypothesis [63].

\section{Additional files}

Additional file 1: Saturation plot of the third codon positions for the Cytochrome B fragment of $\mathbf{3 5}$ Leptodactylinae species.

Transitions (indicated by triangles) and transversions (indicated by X) are plotted against the Tamura-Nei (1993) distance.

Additional file 2: Character states used in evolutionary analyses and respective citation sources.

Additional file 3: Leptodactylinae species sampled and GenBank accession numbers.

Additional file 4: PCR protocols for the amplified fragments.

Additional file 5: State probability of the reconstructed life-history traits at each ancestral node of the $50 \%$ majority-rule consensus cladogram.

\section{Competing interests}

The authors declare that they have no competing interests.

\section{Authors' contribution}

EBP: Conception and design of the work, acquisition, analysis and interpretation of data, writing the manuscript. NMM and RGC: Conception of the work, achievement of financial resources, analysis and interpretation of data, revising the manuscript. NEOM: Acquisition, analysis and interpretation of data. MNCK: Acquisition and interpretation of data. All authors read, reviewed and approved the final manuscript.

\section{Authors' information}

EBP conducted this study as part of her Master in Ecology and Evolution at the Universidade Federal de Goiás under the advisement of NMM and RGC.

\section{Acknowledgments}

This work was supported by competitive grants from CNPq (project no 475333/2011-0) to NMM and from the projects PRONEX CNPq/FAPEG/AUX PESQ CH 007/2009 and FAPDF 193.000.292/2007. EBP and NEOM received scholarships from CNPq and CAPES/MEC, respectively. We thank Marcelo Menin, Albertina Lima and Domingos Rodrigues for providing information on the reproductive biology of Leptodactylinae used in this study, Marion Chartier for providing the R script to generate the pie charts, Fabricio Villalobos, Fausto Nomura and three anonymous referees for helpful comments on the manuscript, and IBAMA/RAN for providing sampling permission (license no. 021010.002153/05-11).

\section{Author details}

'Laboratório de Genética \& Biodiversidade, Instituto de Ciências Biológicas, Universidade Federal de Goiás (UFG), Campus Samambaia, 74001-970 Goiânia, Goiás, Brazil. 'Laboratório de Herpetologia e Comportamento Animal Departamento de Ecologia, Instituto de Ciências Biológicas, Universidade Federal de Goiás, Campus Samambaia, 74001-970 Goiânia, Goiás, Brazil. ${ }^{3}$ Unidade Acadêmica de Ciências Biológicas/CSTR, Universidade Federal de Campina Grande (UFCG), 58704-300 Patos, Paraíba, Brazil.

Received: 29 January 2015 Accepted: 29 April 2015

Published online: 20 May 2015

\section{References}

1. Avise JC. Evolutionary Pathways in Nature. Cambridge: Cambridge University Press; 2006.

2. Schluter D. Ecology and the origin of species. Trends Ecol Evol. 2001;16:372-80.

3. Rundle HD, Nosil P. Ecological speciation. Ecol Lett. 2005;8:336-52.

4. Janzen DH. When is it coevolution? Evolution. 1980;34:611-2.

5. Pagel M. Inferring the historical patterns of biological evolution. Nature. 1999:401:877-84.

6. Nielsen R. Mapping mutations on phylogenies. Syst Biol. 2002;51:729-39.

7. Huelsenbeck JP, Rannala B. Detecting correlation between characters in a comparative analysis with uncertain phylogeny. Evolution. 2003;57:1237-47.

8. Huelsenbeck JP, Nielsen R, Bollback JP. Stochastic mapping of morphological characters. Syst Biol. 2003;52:131-58.

9. Haddad C, Prado C. Reproductive modes in frogs and their unexpected diversity in the Atlantic Forest of Brazil. Bioscience. 2005;55:207-17.

10. Salthe SN, Duellman WE. Quantitative Constrains Associated with Reproductive Mode in Anurans. In: Evolutionary Biology of the Anurans: Contemporary Research on Major Problems, vol. 19. Columbia: University of Missouri Press; 1973. p. 229-49.

11. Pough FH, Janis CM, Heiser JB. A Vida dos Vertebrados. 4th ed. Atheneu Editora: São Paulo; 2008.

12. Duellman WE, Trueb L. Biology of Amphibians. Baltimore, U.S.A.: The Johns Hopkins Univ. Press; 1994.

13. Gomez-Mestre I, Pyron RA, Wiens JJ. Phylogenetic analyses reveal unexpected patterns in the evolution of reproductive modes in frogs. Evolution. 2012;66:3687-700.

14. McDiarmid RW. Evolution of Parental Care in Frogs. In: Burghardt GM, Bekoff M, editors. The Development of Behavior: Comparative and Evolutionary Aspects. New York: Garland STPM Press; 1978. p. 127-47.

15. Heyer WR. The adaptive ecology of the species groups of the genus Leptodactylus (Amphibia, Leptodactylidae). Evolution. 1969;23:421-8.

16. Zimkus BM, Lawson L, Loader SP, Hanken J. Terrestrialization, miniaturization and rates of diversification in African puddle frogs (Anura: Phrynobatrachidae). PLoS One. 2012;7:1-11. 
17. Frost D, Grant T, Faivovich J, Bain R, Haas A, Haddad CFB, et al. The amphibian tree of life. Bull Am Museum Nat Hist. 2006:297:1-370.

18. Pyron RA, Wiens JJ. A large-scale phylogeny of Amphibia including over 2800 species, and a revised classification of extant frogs, salamanders, and caecilians. Mol Phylogenet Evol. 2011;61:543-83.

19. Lavilla EO, Langone JA, Caramaschi U, Heyer WR, De Sá RO. The identification of Rana ocellata Linnaeus, 1758. Nomenclatural impact on the species currently known as Leptodactylus ocellatus (Leptodactylidae) and Osteopilus brunneus (Gosse, 1851) (Hylidae). Zootaxa. 2010;2346:1-16.

20. Heyer WR. Relationship of the marmoratus species group (Amphibia, Leptodactylidae) within the subfamily leptodactylinae. Contrib Sci. 1974:253:1-45

21. Fouquet $A$, Blotto BL, Maronna MM, Verdade VK, Juncá FA, de Sá R, et al. Unexpected phylogenetic positions of the genera Rupirana and Crossodactylodes reveal insights into the biogeography and reproductive evolution of leptodactylid frogs. Mol Phylogenet Evol. 2013;67:445-57.

22. Angulo A, Icochea J. Cryptic species complexes, widespread species and conservation: lessons from Amazonian frogs of the Leptodactylus marmoratus group (Anura: Leptodactylidae). Syst Biodivers. 2010;8:357-70.

23. Fouquet A, Santana Cassini C, Fernando Baptista Haddad C, Pech N, Trefaut Rodrigues M. Species delimitation, patterns of diversification and historical biogeography of the Neotropical frog genus Adenomera (Anura, Leptodactylidae). J Biogeogr. 2013:41:855-70.

24. Duellman WE. Alternative Life-History Styles in Anuran Amphibians: Evolutionary and Ecological Implications. In: Bruton MN, editor. Altern Life-history Styles Anim. Dordrecht: Kluwer Academic Publishers; 1989. p. 101-26.

25. De La Riva I. A new reproductive mode for the genus Adenomera (Amphibia: Anura: Leptodactylidae): taxonomic implications for certain Bolivian and Paraguayan populations. Stud Neotrop Fauna Environ. 1995:30:15-29

26. de Prado CA, Uetanabaro M, Haddad CFB. Description of a new reproductive mode in Leptodactylus (Anura, Leptodactylidae), with a review of the reproductive specialization toward terrestriality in the genus. Copeia. 2002:4:1128-33.

27. Prado CPA, Toledo LF, Zina J, Haddad CFB. Trophic eggs in the foam nests of Leptodactylus labyrinthicus (Anura:Leptodactylidae): an experimental approach. Herpetol J. 2005;15:279-84.

28. Gibson RC, Buley KR. Maternal care and obligatory oophagy in Leptodactylus fallax: a new reproductive mode in frogs. Copeia. 2004;1:128-35.

29. Downie J, Nokhbatolfoghahai M. Presence and absence of the cement gland in foam nesting leptodactylids (Anura: Leptodactylidae): implications for the transition to terrestrial development. Herpetol J. 2006;16:77-81.

30. Faivovich J, Ferraro D, Basso N. A phylogenetic analysis of Pleurodema (Anura: Leptodactylidae: Leiuperinae) based on mitochondrial and nuclear gene sequences, with comments on the evolution. Cladistics. 2012;1:1-23.

31. De Sá RO, Heyer WR, Camargo A. A phylogenetic analysis of Vanzolinius Heyer, 1974 (Amphibia, Anura, Leptodactylidae): taxonomic and life history implications. Arq do Mus Nac Rio Janeiro. 2005;63:707-26.

32. Ponssa ML. Cladistic analysis and osteological descriptions of the frog species in the Leptodactylus fuscus species group (Anura, Leptodactylidae). J Zool Syst Evol Res. 2008;46:249-66.

33. Heyer WR. Systematics of the marmoratus group of the frog genus Leptodactylus (Amphibia, Leptodactylidae). Contrib Sci. 1973:251:1-50.

34. de Almeida AP, Angulo A. A new species of Leptodactylus (Anura: Leptodactylidae) from the state of Espírito Santo, Brazil, with remarks on the systematics of associated populations. Zootaxa. 2006;1334:1-25.

35. Pianka ER. On $r$ and K selection. Am Nat. 1970;104:592-7.

36. Price PW. Strategies for egg production. Evolution. 1974;28:76-84.

37. Clutton-Brock TH. The Evolution of Parental Care. Princeton Univ. Press: Princeton; 1991

38. Heyer WR, McDiarmid RW, Weigmann DL. Tadpoles, predation and pond habitats in the tropics. Biotropica. 1975;7:100-11.

39. Magnusson WE, Hero J-M. Predation and the evolution of complex oviposition behaviour in Amazon rainforest frogs. Oecologia. 1991;86:310-8.

40. Crump ML. Parental care among the amphibia. Adv Study Behav. 1996:25:109-44.

41. Salthe SN, Mecham JS. Reproductive and courtship patterns. In: Physiol Amphib, vol. 59. New York: New York Academic Press; 1974. p. 309-521.

42. Silva FR, Almeida-Neto M, do Prado VHM, Haddad CFB, Rossa-Feres CD. Humidity levels drive reproductive modes and phylogenetic diversity of amphibians in the Brazilian Atlantic Forest. J Biogeogr. 2012;39:1720-32.
43. Müller H, Liedtke HC, Menegon M, Beck J, Ballesteros-Mejia L, Nagel P, et al. Forests as promoters of terrestrial life-history strategies in East African amphibians. Biol Lett. 2013;9:20121146.

44. Heyer WR. The relationships of Leptodactylus diedrus (Anura, Leptodactylidae). Alytes. 1998;16:1-24.

45. Larson PM, de Sá RO. Chondrocranial morphology of Leptodactylus larvae its utility in phylogenetic reconstruction. J Morphol. 1998;305:287-305.

46. de Kokubum MNC, Giaretta AA. Reproductive ecology and behaviour of a species of Adenomera (Anura, Leptodactylinae) with endotrophic tadpoles: systematic implications. J Nat Hist. 2005;39:1745-58.

47. Ponssa M, Jowers M, de Sá R. Osteology, natural history notes, and phylogenetic relationships of the poorly known Caribbean frog Leptodactylus nesiotus (Anura, Leptodactylidae). Zootaxa. 2010;2646:1-25.

48. Maxson LR, Heyer WR. Molecular systematics of the frog genus Leptodactylus (Amphibia: Leptodactylidae). Fieldiana Zoology. 1988;41:1-8.

49. Bossuyt F, Milinkovitch MC. Convergent adaptive radiations in Madagascan and Asian ranid frogs reveal covariation between larval and adult traits. Proc Natl Acad Sci USA. 2000;97:6585-90.

50. Reeder T. Phylogenetic relationships among phrynosomatid lizards as inferred from mitochondrial ribossomal DNA sequences: substitutional bias and information content of transitions relative to transversions. Mol Phylogenet Evol. 1995:4:203-22

51. Moritz C, Schneider C, Wake D. Evolutionary relationships within the Ensatina eschscholtzii complex confirm the ring species interpretation. Syst Biol. 1992;41:273-91

52. Kocher TD, Thomas WK, Meyer A, Edwards SV, Pääbo S, Villablanca FX, et al. Dynamics of mitochondrial DNA evolution in animals: amplification and sequencing with conserved primers. Proc Natl Acad Sci USA. 1989;86:6196-200.

53. Edgar RC. MUSCLE: multiple sequence alignment with improved accuracy and speed. Proc 2004 IEEE Comput Syst Bioinforma Conf. 2004:728-29.

54. Tamura K, Nei M. Estimation of the number of nucleotide substitutions in the control region of mitochondrial DNA in humans and chimpanzees. Mol Biol Evol. 1993:10:512-26.

55. Xia X, Xie Z. DAMBE: software package for data analysis in molecular biology and evolution. J Hered. 2001;92:371-3.

56. Darriba D, Taboada GL, Doallo R, Posada D. jModelTest 2: more models, new heuristics and parallel computing. Nat Methods. 2012:9:772.

57. Ronquist F, Teslenko M, van der Mark P, Ayres DL, Darling A, Höhna S, et al. MrBayes 3.2: efficient Bayesian phylogenetic inference and model choice across a large model space. Syst Biol. 2012;61:539-42.

58. Leaché A, Reeder T. Molecular systematics of the eastern fence lizard (Sceloporus undulatus): a comparison of parsimony, likelihood, and Bayesian approaches. Syst Biol. 2002;51:44-68.

59. Swofford DL. PAUP*. Phylogenetic Analysis Using Parsimony (*and Other Methods). Massachusetts, U.S.A: Sinauer Associates; 2003.

60. Felsenstein J. Phylogenies and the comparative method. Am Nat. 1985;125:1-15.

61. Hillis D, Bull J. An empirical test of bootstrapping as a method for assessing confidence in phylogenetic analysis. Syst Biol. 1993:42:182-92.

62. Bollback JP. SIMMAP: stochastic character mapping of discrete traits on phylogenies. BMC Bioinformatics. 2006;7:88.

63. Pereira EB, Collevatti RG, Kokubum MNC, Miranda NEO, Maciel NM. 1. Table containing the GenBank accession numbers of the sequences from Adenomera, Leptodactylus and Lithodytes used in phylogenetic. 2. Table with the character states for the six life-history traits (reproductive mode, clutch size, habitat, tadpole environment, nuptial pads or spines and egg pigmentation) for 35 Leptodactylinae species. 3. State probability of the six reconstructed life-history traits at each ancestral node of the 50\% majority-rule consensus cladogram. 4. Life-history traits reconstructed were reproductive mode, clutch size, habitat, tadpole environment, nuptial pads or spines and egg pigmentation. 5. Nexus file used in MrBayes software to generate Bayesian molecular phylogenetic hypothesis. Dryad Digital Repository. 2015 http://dx.doi.org/10.5061/dryad.88kj7. 